\section{Exosomes in lung cancer liquid biopsies: Two sides of the same coin?}

The absence of suitable diagnostic, prognostic and predictive biomarkers for non-small cell lung cancer (NSCLC) and the limited efficacy of the available treatments are responsible of the low survival rates of this disease. Conventional chemotherapy, in combination with radiotherapy, has improved outcomes, but a significant proportion of NSCLC patients do not respond to these treatments [1]. The discovery of new molecular targets and drugs, as tyrosine-kinase inhibitors (TKIs) have led to the development of different generations of targeted therapies ameliorating clinical outcomes of the disease. But however, still nowadays there are no definitive treatments for NSCLC and the research of new noninvasive early diagnostic and prognostic biomarkers is mandatory [2]. In this field, liquid biopsies, which include tumor components as circulating tumor cells (CTCs), circulating tumor DNA (ctDNA) and exosomes, could be the long awaited response to this tricky task [3].

Exosomes, one of the lesser-known liquid biopsies components, are cell-derived nanovescicles $(30-100 \mathrm{~nm}$ of diameter) firstly described studying reticulocyte differentiation [4]. Exosomes are formed by the inward budding of multivesicular bodies (MVB), component of the endocytic pathway [5]. Exosomes are released from different types of cells both under physiological and pathological conditions and, interestingly, have been well detected in several body fluids, such as peripheral blood, urine, pleural effusion, amniotic fluid, saliva, breast-milk and cerebrospinal fluid $[3,6]$. They function as messenger particles, playing a role in cell-to-cell communication and to deliver proteins, lipids, mRNAs and miRNAs with biological activity in the target cells [3]. Now it is well known that these vesicles have pleiotropic functions in remodeling the tumor microenvironment, promoting immune escape and in tumor progression [7].

In the last years, the molecular profiling of these nanovesicles in the plasma of patients with NSCLC, offered the opportunity to delineate better the characteristics of the disease. Exosomes released from NSCLC patients contain proteins related to tumor growth and progression, such as EGFR, claudins, K-ras and inducers of matrix metalloproteinase synthesis like EMMPRIN [8]. Similarly to these findings, in other cancer types several exosomal proteins have been described with diagnostic and prognostic features, such as MIA and S100B in melanoma and Glypican- 1 in early pancreatic cancer $[9,10]$. So, these features might be exploited as a source of specific biomarkers because they reflect the pathological features of the disorder. Jakobsen et al. demonstrated the potential diagnostic properties of exosomal proteins in advanced NSCLC. Exosomes isolated from plasma of 109 NSCLC patients (stage IIIa - IV) and 110 healthy controls were analyzed with an extracellular vesicle array that phenotyped the exosomes directly from the plasma samples using 37 antibodies against lung cancer-related proteins, initiating the way to generate minimally invasive tools to detect exosome-derived biomarkers [11]. In this context, plasma from 431 lung cancer patients were analyzed in order to identify potential diagnosis proteins and demonstrating a protein profile associated to adenocarcinoma [12]. Other exosome's features suitable to be biomarkers have been also demonstrated in studies of other body fluids, such as urine. For example, Li et al., in NSCLC patients demonstrated a positive correlation rate of $65 \%$ between the presence of leucine-rich $\alpha$-2-glycoprotein (LRG1) measured in primary tumor and in urinary exosomes. This study indicates that LRG1 in urinary exosomes might be derived from primary tumor tissue and, then, could be suitable as a non-invasive diagnostic biomarker of NSCLC in this selected population [13]. In preclinical analysis with NSCLC cell lines, a proteomic profiling was performed in exosomes identifying a total of 721 proteins. Among these, several proteins such as EGFR, SRC and GRB2, were found really enriched, suggesting then their potential prognostic biomarker role in NSCLC patients [14]. Recently, it has been described for the first time the translocation ALK-EML4 inside exosomes from NSCLC patients, broadening exosome characterization as a powerful tool for molecular identification [15].

Exosomes have been described also as delivery systems, specifically, exosomes derived from dendritic cells (DEX) have been studied as possible immunotherapy components since they carry major histocompatibility complex (MHC) molecules and are able to present antigens to immune cells as CD4 and CD8T lymphocytes [16]. A phase I and a phase II trial have been performed with DEX, concluding in both cases the safety of the administration of DEX and DEX carrying IL-15Ra and NKG2D and the ability of DEX to activate both innate and adaptive immune systems $[17,18]$.

In addition to their effects on the primary tumor microenvironment, exosomes also play a strong role, together with chemokines, in the establishment of the pre-metastatic niche. In SCID mice it has been demonstrated that there is a lung pre-metastatic niche formation mediated also by CD105-positive exosomes through an upregulation of proteins such as VEGFR1, MMP2 and MMP9 [19]. In other preclinical studies, exosomes were also described as potential drug resistance vectors. Xiao et al. demonstrated in vitro that exosomes released by A549 cells were involved in the regulation of the sensitivity of these cells to DNA-damaging Platinum (DDP) Furthermore, exosomes released by A549 cells during DDP exposure decreased the sensitivity of other untreated A549 cells to DDP, which may be mediated by exosome-mediated proteins, miRNAs and mRNAs cell exchange [20].

The characterization of tissular miRNA profiles in NSCLC patient's $[21,22]$ prompts the idea that exosomal miRNA profile could also mirror the tumoral tissue profile, and serve as a specific liquid biopsy biomarker [23]. In this regards, Rabinowits et al., for the first time, demonstrated that in lung adenocarcinoma patients there is a similarity between exosomal miRNAs and tumor-derived miRNAs profiles, suggesting that 12 specific exosomal miRNAs 
(miR-17-3p, miR-21, miR-106a, miR-146, miR-155, miR-191, miR192, miR-203, miR-205, miR-210, miR-212, and miR-214) might be useful as screening biomarkers for lung adenocarcinoma [24]. Recent studies have also been performed in order to understand the role of miRNAs in exosomes released in other body fluids. Rodriguez et al. performed a prospective exosomal analysis in blood and bronchoalveolar lavage (BAL) samples from 30 NSCLC patients and 75 controls [25]. The results showed that increased amounts of exosomes were detected in plasma compared than in BAL samples in both groups. Additionally, miR-302-a and miR-302-c were detected specifically in BAL and miR-126 in plasma, suggesting a selective tumor-derived exosome destination and a selected exosomal disposal of miRNAs in NSCLC patients.

Exosomes are important components of the liquid biopsy, that contain proteins and nucleic acids that permit to get a specific tumor molecular profile, and may act as a surrogate tumor-ID-card. This could help in establishing non-invasive diagnostic, prognostic and drug resistance biomarkers that could be crucial for managing NSCLC patients. Additional studies are mandatory in order to translate these discoveries to the clinical setting, especially in the standardization of the exosome isolation and characterization methods, which they are still debated.

\section{Conflict of interests}

None.

\section{References}

[1] B. Bhardwaj, S. Revannasiddaiah, H. Bhardwaj, S. Balusu, A. Shwaiki, Molecular targeted therapy to improve radiotherapeutic outcomes for non-small cell lung carcinoma, Ann. Transl. Med. 4 (2016) 50, http://dx.doi. org/10.3978/j.issn.2305-5839.2015.10.35.

[2] P.A.J. Crosbie, R. Shah, Y. Summers, C. Dive, F. Blackhall, Prognostic and predictive biomarkers in early stage NSCLC: CTCs and serum/plasma markers, Transl. Lung Cancer Res. 2 (2013) 382-397, http://dx.doi.org/10.3978/j.issn. 2218-6751.2013.09.02.

[3] C. Rolfo, M. Castiglia, D. Hong, R. Alessandro, I. Mertens, G. Baggerman, et al., Liquid biopsies in lung cancer: the new ambrosia of researchers, Biochim. Biophys. Acta - Rev. Cancer 1846 (2014) 539-546, http://dx.doi.org/10.1016/j. bbcan.2014.10.001.

[4] R.M. Johnstone, M. Adam, J.R. Hammond, L. Orr, C. Turbide, Vesicle formation during reticulocyte maturation. Association of plasma membrane activities with released vesicles (exosomes), J. Biol. Chem. 262 (1987) 9412-9420.

[5] J. Kowal, M. Tkach, C. Théry, Biogenesis and secretion of exosomes, Curr. Opin. Cell Biol. 29 (2014) 116-125 http://dx.doi.org/10.1016/j.ceb.2014.05.004.

[6] C. Corrado, S. Raimondo, A. Chiesi, F. Ciccia, G. De Leo, R. Alessandro, Exosomes as intercellular signaling organelles involved in health and disease: basic science and clinical applications, Int. J. Mol. Sci. 14 (2013) 5338-5366, http://dx.doi.org/10.3390/ijms14035338.

[7] S. Fontana, L. Saieva, S. Taverna, R. Alessandro, Contribution of proteomics to understanding the role of tumor-derived exosomes in cancer progression: state of the art and new perspectives, Proteomics 13 (2013) 1581-1594, http://dx.doi.org/10.1002/pmic.201200398.

[8] S.-H. Huang, Y. Li, J. Zhang, J. Rong, S. Ye, Epidermal growth factor receptor-containing exosomes induce tumor-specific regulatory $\mathrm{T}$ cells, Cancer Invest. 31 (2013) 330-345, http://dx.doi.org/10.3109/07357907.2013. 789905.

[9] E. Alegre, L. Zubiri, J.L. Perez-Gracia, M. González-Cao, L. Soria, S. Martín-Algarra, et al., Circulating melanoma exosomes as diagnostic and prognosis biomarkers, Clin. Chim. Acta 454 (2016) 28-32, http://dx.doi.org/ 10.1016/j.cca.2015.12.031.

[10] S. a. Melo, L.B. Luecke, C. Kahlert, A.F. Fernandez, S.T. Gammon, J. Kaye, et al., Glypican-1 identifies cancer exosomes and detects early pancreatic cancer, Nature 523 (2015) 177-182, http://dx.doi.org/10.1038/nature14581.

[11] K.R. Jakobsen, B.S. Paulsen, R. Baek, K. Varming, B.S. Sorensen, M.M. Jørgensen, Exosomal proteins as potential diagnostic markers in advanced non-small cell lung carcinoma, J. Extracell. Vesicles 4 (2015) 26659, http://dx.doi.org/10. 3402/jev.v4.26659.

[12] B. Sandfeld-Paulsen, K.R. Jakobsen, R. Bæk, B.H. Folkersen, T.R. Rasmussen, P. Meldgaard, et al., Exosomal proteins as a diagnostic biomarkers in lung cancer, J. Thorac. Oncol. 0 (2016) 1462-1474, http://dx.doi.org/10.1016/j.jtho. 2016.05.034.
[13] Y. Li, Y. Zhang, F. Qiu, Z. Qiu, Proteomic identification of exosomal LRG1: A potential urinary biomarker for detecting NSCLC, Electrophoresis 32 (2011) 1976-1983, http://dx.doi.org/10.1002/elps.201000598.

[14] D.J. Clark, W.E. Fondrie, A. Yang, L. Mao, Triple SILAC quantitative proteomic analysis reveals differential abundance of cell signaling proteins between normal and lung cancer-derived exosomes, J. Proteomics (2015), http://dx. doi.org/10.1016/j.jprot.2015.12.023.

[15] K. Brinkmann, D. Enderle, T. Koestler, S. Bentink, J. Emenegger, A. Spiel, et al. Abstract 545: Plasma-based diagnostics for detection of EML4-ALK fusion transcripts in NSCLC patients, Cancer Res. 75 (2015), http://dx.doi.org/10. 1158/1538-7445.AM2015-545, 545-545.

[16] S. Hiltbrunner, P. Larssen, M. Eldh, A.K. Wagner, M.C.I. Karlsson, S. Gabrielsson, Exosomal cancer immunotherapy is independent of MHC molecules on exosomes, Oncotarget 7 (2016), http://dx.doi.org/10.18632/oncotarget.9585.

[17] M.A. Morse, J. Garst, T. Osada, S. Khan, A. Hobeika, T.M. Clay, et al., A phase I study of dexosome immunotherapy in patients with advanced non-small cell lung cancer, J. Transl. Med. 3 (2005) 1-8, http://dx.doi.org/10.1186/14795876-3-9.

[18] S. Viaud, C. Thery, S. Ploix, T. Tursz, V. Lapierre, O. Lantz, et al., Dendritic cell-derived exosomes for cancer immunotherapy: what's next? Cancer Res. 70 (2010) 1281-1285, http://dx.doi.org/10.1158/0008-5472.CAN-09-3276.

[19] C. Grange, M. Tapparo, F. Collino, L. Vitillo, C. Damasco, M.C. Deregibus, et al., Microvesicles released from human renal cancer stem cells stimulate angiogenesis and formation of lung premetastatic niche, Cancer Res. 71 (2011) 5346-5356, http://dx.doi.org/10.1158/0008-5472.CAN-11-0241.

[20] X. Xiao, S. Yu, S. Li, J. Wu, R. Ma, H. Cao, et al., Exosomes: decreased sensitivity of lung cancer A549 cells to cisplatin, PLoS One 9 (2014) 1-6, http://dx.doi. org/10.1371/journal.pone.0089534.

[21] N. Yanaihara, N. Caplen, E. Bowman, M. Seike, K. Kumamoto, M. Yi, et al., Unique microRNA molecular profiles in lung cancer diagnosis and prognosis, Cancer Cell 9 (2006) 189-198, http://dx.doi.org/10.1016/j.ccr.2006.01.025.

[22] M. Garofalo, C. Quintavalle, G. Di Leva, C. Zanca, G. Romano, C. Taccioli, et al. MicroRNA signatures of TRAIL resistance in human non-small cell lung cancer, Oncogene 27 (2008) 3845-3855, http://dx.doi.org/10.1038/onc.2008.6.

[23] R. Munagala, F. Aqil, R. Gupta, Exosomal miRNAs as biomarkers of recurrent lung cancer, Tumor Biol. 37 (2016) 10703-10714, http://dx.doi.org/10.1007/ s13277-016-4939-8.

[24] G. Rabinowits, C. Gerçel-Taylor, J.M. Day, D.D. Taylor, G.H. Kloecker, Exosomal MicroRNA: A diagnostic marker for lung cancer, Clin. Lung Cancer 10 (2016) 42-46, http://dx.doi.org/10.3816/CLC.2009. n.006.

[25] M. Rodríguez, J. Silva, A. López-Alfonso, M. López-Muñiz, C. Peña, G. Domínguez, et al., Different exosome cargo from plasma/bronchoalveolar lavage in non-small-cell lung cancer, Genes Chromosomes Cancer 53 (2014) 713-714, http://dx.doi.org/10.1002/gcc.22181.

\author{
Christian Rolfo* \\ Marco Giallombardo \\ Pablo Reclusa \\ Rafael Sirera \\ Marc Peeters
}

Phase I - Early Clinical Trials Unit,Oncology Department, Antwerp University Hospital E Center for Oncological Research (CORE) Antwerp University, Antwerp, Belgium

* Corrsponding author at: Head of Phase I - Early Clinical Trials Unit, Oncology Department Antwerp University Hospital. Wilrijkstraat 10, 2650 Edegem, Belgium.

E-mail address: christian.rolfo@uza.be (C. Rolfo)

26 July 2016

10 November 2016

17 November 2016 